\title{
GOLPH2-regulated oncolytic adenovirus, GD55, exerts strong killing effect on human prostate cancer stem-like cells in vitro and in vivo
}

Chang YING ${ }^{1, \#}$, Bo-duan XIAO ${ }^{1, \#}$, Yun QIN ${ }^{1}$, Bin-rong WANG ${ }^{1}$, Xin-yuan LIU $^{1,2}$, Ru-wei WANG ${ }^{3}$, Ling FANG ${ }^{3}$, Hui YAN ${ }^{4}$, Xiu-mei ZHOU ${ }^{1}$, Yi-gang WANG ${ }^{1, *}$

${ }^{1}$ Xinyuan Institute of Medicine and Biotechnology, College of Life Sciences Zhejiang Sci-Tech University, Hangzhou 310018, China; ${ }^{2}$ Institute of Biochemistry and Cell Biology, Shanghai Institutes for Biological Sciences, Chinese Academy of Sciences, Shanghai 200031, China; ${ }^{3}$ Zhejiang Conba Pharmaceutical Co., Ltd, Hangzhou 310018, China; ${ }^{4}$ Zhejiang Academy of Medical Sciences, Hangzhou 310013, China

\begin{abstract}
GOLPH2 (also called GP73) is a Golgi glycoprotein, which has been identified as a novel tumor marker upregulated in various cancers, including prostate cancer (PCa). GD55 is a novel GOLPH2-regulated oncolytic adenovirus that exhibits a strong killing effect on hepatoma cells. Here, we investigate the antitumor effect of GD55 on prostate cancer stem cell (CSC)-like cells in vitro and in vivo. Prostate CSC-like sphere cells were acquired and enriched by culturing DU145, LNCap or P3 prostate cancer cells in suspension. The prostate CSC-like sphere cells were capable of self-renewal, differentiation and quiescence, displaying tumorigenic feature and chemo-resistance to 5-FU, doxorubicin and DDP. Treatment with GD55 (1, 5, $10 \mathrm{MOI}$ ) dose-dependently suppressed the viability of DU145 sphere cells, which was a more pronounced compared to its cytotoxic action on the parental DU145 cells. In a mouse xenograft prostate CSC-like model, intratumoral injection of GD55 markedly suppressed the growth rate of xenograft tumors and induced higher levels of cell death and necrosis within the tumor tissues. Our results demonstrate that GD55 infection exerts strong anticancer effects on prostate CSC-like cells in vitro and in vivo, and has a potential to be used in the clinical therapy of PCa.
\end{abstract}

Keywords: prostate cancer; cancer stem cells; GOLPH2; oncolytic adenovirus; GD55; antitumor agents; cancer targeting genevirotherapy

Acta Pharmacologica Sinica (2018) 39: 405-414; doi: 10.1038/aps.2017.91; published online 7 Sep 2017

\section{Introduction}

Prostate cancers (Pca) are common malignant tumors of the male urinary system, and they can easily develop into hormone-refractory prostate cancer, which renders them difficult to cure. Long-term studies have shown that the recurrence rate of primary prostate cancer in patients within 10 years is as high as $30 \%^{[1]}$. Total androgen blockade is the main treatment approach for localized prostate cancer, but this can lead to the emergence of androgen-independent cells and has thus become one of the greatest challenges in the treatment of this disease $^{[2]}$. Thus, the traditional therapeutic methods are not always applicable, as it is arduous to achieve the desired ther-

\footnotetext{
\# These authors contributed equally to this work.

${ }^{*}$ To whom correspondence should be addressed.

E-mail wangyigang43@163.com

Received 2017-03-28 Accepted 2017-06-05
}

apeutic efficacy for advanced or metastatic cancer. Therefore, it is urgent to explore novel treatment strategies to improve therapy to ensure better survival and quality of life in PCa patients.

The cancer stem cell (CSC) model provides insights into the mechanisms behind tumor initiation, progression, metastasis, and recurrence, which result from cellular processes that contribute to phenotypic and functional heterogeneity in several tumors $^{[3,4]}$. The current hypothesis is that CSCs are located at the top of the cellular hierarchy and give rise to new differentiated non-CSC lineages. In this regard, CSCs are thought to drive tumor growth and disease recurrence by promoting metastasis and resistance to therapy ${ }^{[5]}$. Therefore, CSCs are one of the primary determinants of metastatic dissemination and disease relapse in the course of cancer treatments, and they could be used as critical targets to eliminate different types of cancer, including prostate cancer ${ }^{[6]}$.

Recent efforts to construct oncolytic adenoviruses to target 
and kill cancer cells have been successful, in particular, for the treatment of drug-resistant human cancers. So far, many targeted strategies based on oncolytic adenoviruses have been designed and reported to show potent antitumor activity in various preclinical studies ${ }^{[7,8]}$. Moreover, oncolytic viruses targeting CSCs are not restricted to enable the understanding of the mechanisms of drug resistance encountered in traditional treatments ${ }^{[9]}$. Oncolytic adenoviruses can efficiently infect both highly proliferative cells (non-CSCs) and quiescent cells (CSCs), without affecting cells through the ATP-binding cassette $(A B C)$ transporters as seen in the case of chemotherapeutic drugs ${ }^{[10-12]}$.

The previously designed cancer targeting gene-virotherapy strategy (CTGVT) from our laboratory, which integrates the advantages of gene therapy with virotherapy, may be a novel method of tumor treatment ${ }^{[8,13]}$. Based on the construction of CTGVT, the oncolytic adenovirus system, ZD55 (ZD55gene) was constructed by deleting the adenoviral E1B55-kD viral protein component $t^{[14]}$. Preclinical data have shown that the ZD55 gene exhibits powerful antitumor effects on various types of cancer models, such as liver cancer, colon cancer, and lung cancer, through the oncolytic action of the virus itself and by increasing the expression of the carried antitumor gene ${ }^{[12,}$ 15]. ZD55, however, lacks targeting ability for specific tumor types, such as prostate cancer. Fortunately, a recent study demonstrated that GOLPH2 is an excellent candidate marker for prostate cancer. Currently, we have engineered a novel oncolytic adenovirus called GD55, which is based on ZD55 and which is regulated by GOLPH2, which has been shown to be a valuable agent for the treatment of liver cancer ${ }^{[16]}$ and liver CSC-like cells ${ }^{[17]}$.

Our present results indicate that GD55 could significantly elicit cytotoxic effects on prostate CSC-like cells and could exhibit a stronger oncolytic effect compared with the common oncolytic virus ZD55. Our present study has successfully demonstrated that GD55 can effectively destroy prostate CSCs.

\section{Materials and methods}

\section{Cells line, sphere culture and sphere passage}

The human embryonic kidney cell line HEK293 was obtained from Microbix Biosystems Inc (Toronto, Ontario, Canada). Human prostate cancer cell lines DU145, LNCaP and PC3, and the human normal prostate cell line Wpmy-1, were acquired from Shanghai Cell Collection (Shanghai, China) and maintained as a monolayer in RPMI-1640 supplemented with 10\% fetal bovine serum (Gibco, USA), $100 \mathrm{IU} / \mathrm{mL}$ of penicillin, and $100 \mu \mathrm{g} / \mathrm{mL}$ of streptomycin. All cells were cultured in a $5 \%$ $\mathrm{CO}_{2}$-containing humidified incubator at $37^{\circ} \mathrm{C}$.

DU145, LNCaP and PC3 cells were collected and washed to remove serum and then suspended in serum-free DMEM/ F12 (Gibco, USA) supplemented with $20 \mathrm{ng} / \mathrm{mL}$ recombinant human basic fibroblast growth factor (bFGF), $20 \mathrm{ng} / \mathrm{mL}$ recombinant human epidermal growth factor (EGF), $1 \times$ B27, $100 \mathrm{IU} / \mathrm{mL}$ of penicillin and $100 \mu \mathrm{g} / \mathrm{mL}$ of streptomycin in ultra-low attachment 6-well plates (Corning). Fresh DMEM/
F12 with growth factor was added to the 6-well plates every 2 days. Four days later, the tumor spheres were collected by gentle centrifugation and then digested with Accutase (Sigma, USA) into single-cell suspensions for succeeding experiments.

The tumorspheres were digested into single cells, centrifuged to remove the enzyme and then resuspended with conditioned medium in ultra-low attachment 6-well plates to allow the formation of spheres. The spheres were passaged every 4 days.

\section{Generation, purification and titration of adenovirus}

The oncolytic adenoviruses ZD55 and GD55 were previously constructed and stored in our laboratory, and they were not contaminated with wild-type viral strains. GD55 was constructed using the GOLPH2 promoter to replace the ZD55 viral promoter of the E1A gene. The ZD55 and GD55 viral strains were amplified in HEK293 cells. The viral particles were collected by $\mathrm{CsCl}$ gradient centrifugation of the cells, followed by dialysis. Viral titer was detected using TCID50 in HEK293 cells.

\section{Colony formation}

DU145 cells and DU145 sphere cells were digested into single cells and then seeded in RPMI-1640 with $10 \%$ FBS at a density of 1000 cells/well in 6-well plates. After 7 days, the cells were fixed in $4 \%$ paraformaldehyde and stained with crystal violet. The number of colonies with more than 70 cells was quantified.

\section{Cytotoxicity assay}

Cells were seeded at a density of 5000 cells/well into 96-well plates and cultured for $12 \mathrm{~h}$ at $37^{\circ} \mathrm{C}$ prior to treatment with virus (ZD55 or GD55) or drug (Doxorubicin, Cisplatin or 5-Fu). Then, MTT (3-(4,5-dimethylthiazol-2-yl)-2,5-diphenyltetrazolium bromide) solution in PBS $(5 \mathrm{mg} / \mathrm{mL}, 20 \mu \mathrm{L})$ was added into each well. After incubating the cells at $37^{\circ} \mathrm{C}$ for $4 \mathrm{~h}$, the supernatant was removed, and $150 \mu \mathrm{L}$ dimethyl sulfoxide was added to each well. Cell viability was assessed by measuring the absorbance at $490 \mathrm{~nm}$ with a microplate reader after thorough mixing.

\section{Cytopathic assay}

DU145 sphere cells were grown in a 48-well plate and then infected with ZD55 or GD55 at the indicated MOIs; uninfected cells served as the controls. Two days later, the cells were stained with $2 \%$ crystal violet in $20 \%$ methanol for 15 min and then washed using distilled water and documented by photography.

\section{Quantitative RT-PCR (qRT-PCR) assay}

Total RNA was extracted using TRIzol (Invitrogen, Carlsbad, CA, USA) according to the manufacturer's protocol, and cDNA was synthesized with the ReverTra Ace qPCR RT Kit (Toyobo Osaka, Japan). Expression levels of genes were measured with SYBR Green Real-time PCR Master Mix (Toyobo) with specific primers. GAPDH gene was used as an internal 
$\operatorname{control}^{[18]}$.

\section{Western blot analysis}

Total protein was extracted from treated cells using IP lysis buffer. Protein was then separated using SDS-PAGE and electrotransferred to PVDF membranes. Primary antibodies against GOLPH2, XIAP, Bax, Survivin, CD133, CD44, ERK, $\beta$-Catenin, $\mathrm{p}-\mathrm{AKT}, \mathrm{PARP}$ and GAPDH were purchased from Cell Signaling Technology. Primary antibodies against E1A and caspase3 were purchased from Santa Cruz Biotechnology. Antibody against SOX2 was purchased from Antibody Revolution. All secondary antibodies were purchased from MultiSciences.

\section{Hoechst 33342 staining}

DU145 sphere cells were cultured in 6-well plates and then infected with ZD55 or GD55 at the indicated MOIs; uninfected cells served as the controls. After $48 \mathrm{~h}$, cells were stained with Hoechst 33342 (Beyotime, Shanghai, China) at $1 \mathrm{mg} / \mathrm{mL}$ for $30 \mathrm{~min}$ at $37^{\circ} \mathrm{C}$ and observed under an inverted fluorescence microscope.

\section{Flow cytometric analysis}

Cell cycle distribution was analyzed using propidium iodide staining. Cells were collected and washed once with cold PBS. After fixation in $70 \%$ ethanol overnight at $-20^{\circ} \mathrm{C}$, the cells were washed once with cold PBS and then stained with PI (Beyotime Biotech, China) at $37^{\circ} \mathrm{C}$ for $30 \mathrm{~min}$. Fluorescence-activated cell sorting (FACS, Becton Dickinson) was performed immediately after staining.

DU145 sphere cells were grown in 6-well plates and then infected with ZD55 or GD55 at the indicated MOIs. After $48 \mathrm{~h}$, the cells were trypsinized and harvested. Then, the cells were detected using Annexin-V-Fluorescein isothiocyanate (FITC) and propidium iodide (PI) double staining following the manufacturer's instructions. Cell apoptosis was detected using fluorescence-activated cell sorting (BD Biosciences, San Jose, CA, USA).

\section{Animal experiments}

All applicable international, national, and/or institutional guidelines for the care and use of animals were followed. For the tumorigenicity assay, four-week-old female BALB/c nude mice were purchased from the Animal Research Committee of the Institute of Biochemistry and Cell Biology (Shanghai, China). DU145 parental and sphere cells were resuspended at $5 \times 10^{5}$ cells $/ 100 \mu \mathrm{L}$ and subcutaneously injected into the left flanks of each mouse in serum-free DMEM/Matrigel (1:1) using $100 \mu \mathrm{L}$ microsyringes. Each group had 8 mice.

To observe the antitumor effect of adenoviruses against xenografts formed from DU145 spheres, $1.5 \times 10^{6}$ DU145 sphere cells in serum-free DMEM/Matrigel (2:1) were subcutaneously injected into the left flank of each 4-week-old female BALB/ c nude mice ( 8 mice per group). When the xenograft tumors reached $100 \mathrm{~mm}^{3}$, the mice were randomly divided into three groups ( 8 nude mice per group), and a total of $1 \times 10^{9}$ plaque- forming units (PFU) of ZD55, GD55 or PBS were administered per mouse via intratumoral injection once every other day for a total of three times. The tumor volume was measured every 3 days and calculated as length $\times$ width $\times$ width $/ 2$.

\section{Histopathology, IHC and TUNEL assay}

Liver samples and tumor tissues were harvested from mice and fixed in 5\% paraformaldehyde, dehydrated with an increasing gradient of ethanol and embedded in paraffin wax, and cut into $5 \mu \mathrm{m}$ sections. The sections were stained with hematoxylin and eosin for histological analysis. The sections were then incubated in anti-GOLPH2, anti-CD44, and antiNanog antibodies, followed by avidin-biotin-peroxidase complex reagents (Vector Laboratories, Burlingame, CA, USA) for IHC analysis. Hematoxylin was used as a counterstain. The In Situ Apoptosis Detection Kit (Sino-American Biotechnology Co, Luoyang, China) was used to stain apoptotic cells in tumor tissue sections according to the manufacturer's instructions. All sections were counterstained with hematoxylin.

\section{Statistical analysis}

The data are represented as the mean \pm SD. Differences among different treatment groups were assessed using analysis of variance and Student's $t$ test. Differences were considered statistically significant when $P<0.05\left(^{*}\right)$, to be very significant when $\left.P<0.01{ }^{* *}\right)$.

\section{Results}

PCa-specific expression of the GOLPH2 gene and the tumorkilling effect of GOLPH2-regulated GD55 on prostate cell lines

The expression of the GOLPH2 gene was detected in the normal prostate cell line Wpmy-1 and the prostate cancer cell lines DU145, LNCaP and PC3 by qRT-PCR. We found that the expression of the GOLPH2 gene was noticeably higher in the PCa cell lines than in the normal cells (Figure 1A). The PCa cell-specific expression of the GOLPH2 protein was detected by Western blotting (Figure 1B). These results showed that GOLPH2 and p-Akt have high expression and the tumor suppresser p53 has low expression in prostate cancer cells. Further, we investigated the tumor-suppressive activity of GOLPH2-regulated GD55 in vitro using MTT assay. The three prostate cancer cell lines and the normal cell line Wpmy-1 were infected with GD55 and ZD55 at various MOIs. Our cell viability data showed that GD55 had a stronger antitumor effect on PCa cells than did ZD55 in a dose-dependent manner (Figure 1C). The cell growth suppressive rate of GD55 reached approximately $77.43 \%$ in DU145 cells. One limitation with our new GD55 system is its mild cytotoxic effect on normal prostate cells. Collectively, our present results demonstrate that GOLPH2-regulated oncolytic adenovirus GD55 exhibits greater superiority to the typical oncolytic adenovirus ZD55 in eliminating prostate cancer cells in vitro.

\section{Prostate cancer cell lines cultured in suspension acquire CSC-like} characteristics

Suspension culture in growth factor-defined serum-free 

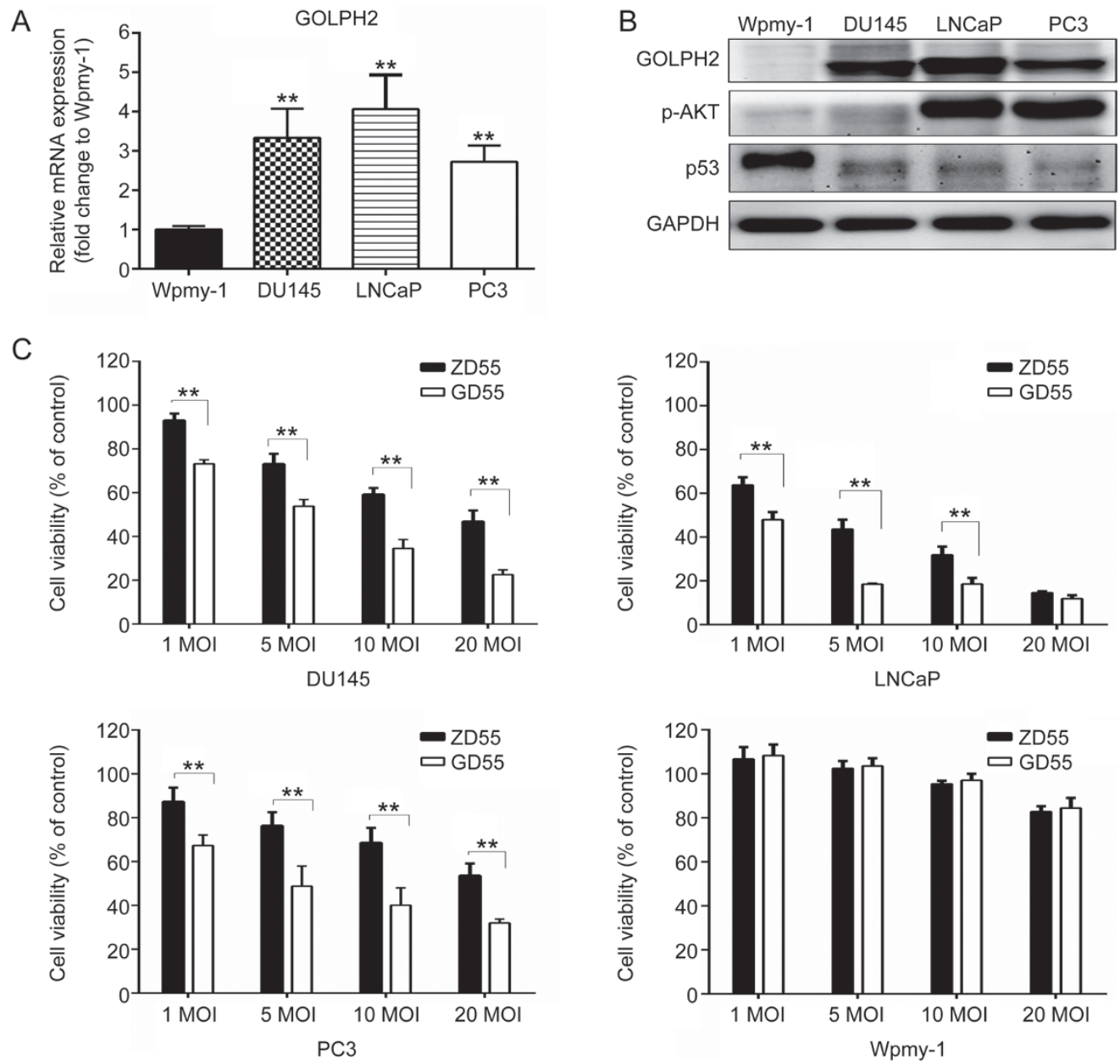

Figure 1. Expression of the GOLPH2 promoter and the tumor-killing effect of GP73-regulated GD55 in prostate cell lines. (A) The activity of the GOLPH2 promoter in three prostate cancer cell lines DU145, LNCaP and PC3 and a normal prostate cell line Wpmy-1 was detected using qRT-PCR. (B) The expression of GOLPH2 protein was detected using Western blotting analysis in three PCa cell lines and one normal prostate cell line. (C) The PCa cell lines DU145, LNCaP, and PC3 and the normal prostate cell line Wpmy-1 were infected with GD55 or ZD55 at MOls of 1, 5, 10, and 20. After 48 h, the cell survival rate was measured via the MTT assay. The results are presented as the mean \pm SD $(n=6)$ and expressed as the percentage relative to mocktreated cells. ${ }^{* *} P<0.01$.

medium can screen and enrich for cells with CSC-like traits ${ }^{[19-21]}$. To examine whether prostate cancer cell lines could acquire CSC-like traits, the suspension cultures of DU145, $\mathrm{LNCaP}$, and $\mathrm{PC} 3$ were grown in specific serum-free medium as described in Materials and Methods. In ultra-low attachment plates, these three cell lines gradually formed non-adherent spheroid bodies over a period of 4-6 days (Supplementary Figure S1A), which we named sphere cells in relation to their parental cells, which were grown as single layers in adherent cultures. As anticipated, the expression of multiple prostate CSC-associated genes (such as CD133 ${ }^{[22,23]}$ and CD44 ${ }^{[24,25]}$ ), which are related to self-renewal, and surface antigen markers of prostate CSCs increased in DU145 sphere cells compared with their parental counterparts (Figure 2B). DU145 sphere cells that expanded in suspension culture for 3 passages were capable of maintaining even higher levels of mRNA expression of CD44, CD133, NANOG, and SOX2 (Supplementary Figure S1B). However, the DU145 sphere cells could gradu- ally revert to the phenotypes of their parental counterparts by the $6^{\text {th }}$ day after being cultured under adherent conditions in serum-containing medium (Supplementary Figure S1C), but some differences remained. Using Western blotting, we further verified that some of the related proteins encoded by the genes mentioned above were upregulated in prostate CSCs and DU145 sphere cells. The anti-apoptosis proteins XIAP and Survivin were also upregulated in DU145 sphere cells (Figure 2A). In principle, the elevated expression patterns of a variety of pleiotropic factors that act as transcription factors (NANOG and OCT4) in CSCs should correspond to equivalent levels of activated signaling circuits in which the related signaling molecules undergo post-translational modification via phosphorylation to transmit signals to induce their transcription. It was observed that DU145 sphere cells expressed higher levels of phosphorylated AKT than the corresponding parental cells (Figure 2A). Cell proliferation assay was carried out by measuring the rate of colony formation in the sphere and parental 
A

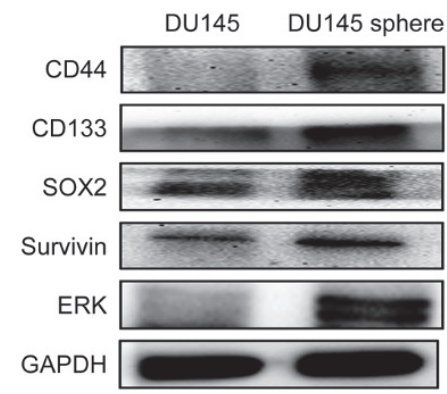

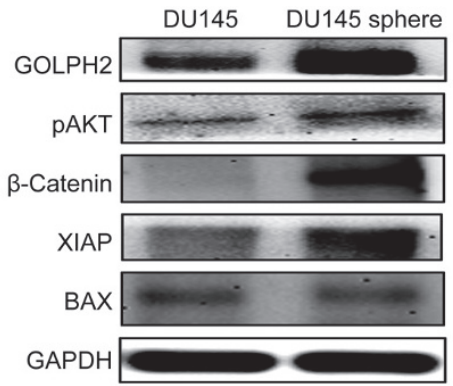

GAPDH

\section{B}

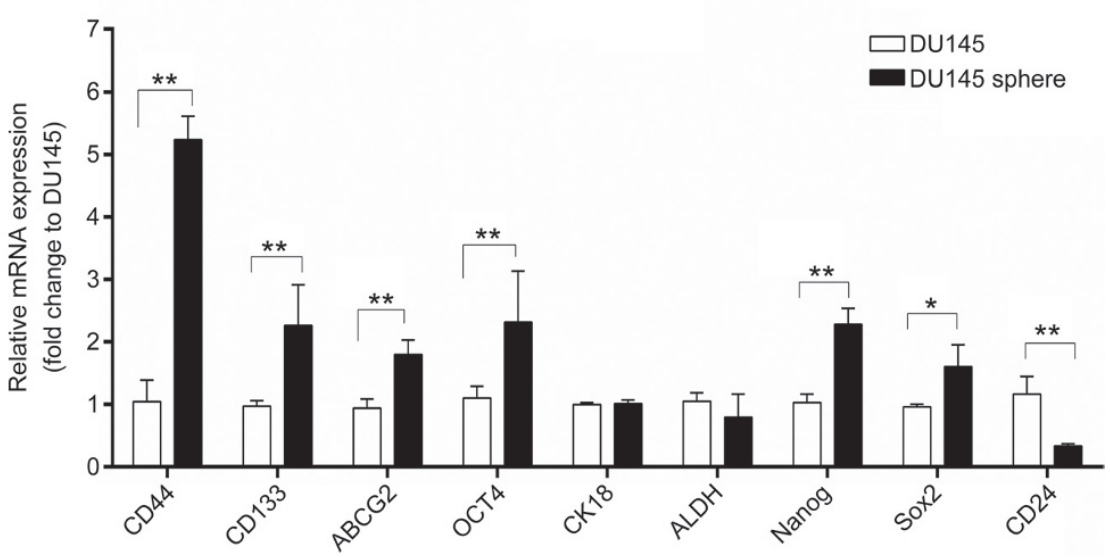

Figure 2. DU145 sphere cells possess multiple properties associated with prostate CSCs. (A) The protein levels of prostate CSC-associated genes and anti-apoptosis genes in DU145 parental and sphere cells are markedly different. (B) DU145 sphere cells show higher expression of prostate CSCassociated genes (eg, CD44, CD133 and Nanog) when compared with DU145 parental cells. The qRT-PCR data were normalized to the GAPDH gene and are shown as fold change relative to DU145 cells. All experiments were repeated three times, and all data shown represent the mean \pm SD ( $n=6)$. ${ }^{*} P<0.05,{ }^{* *} P<0.01$.

DU145 cells. The assay was performed to compare the relative extent of quiescence in both cell types. The results showed that sphere cells proliferated at a lower rate than the parental cells (Supplementary Figure S2A). Additionally, the FACS analysis data showed that the proportion of quiescent cells (in $\mathrm{G}_{0} / \mathrm{G}_{1}$ phase) increased in DU145 sphere cells (Supplementary Figure S2B), compared with that in the parent cells.

CSCs are defined functionally by their robust ability to initiate new tumors in vivo. Various quantities of DU145 sphere cells and parental cells were subcutaneously injected into the left dorsal flanks of BALB/c mice to generate experimental and control groups, respectively, for the evaluation of tumorigenicity. As anticipated, DU145 sphere cells demonstrated significantly higher tumor initiating ability and proliferative rate than parental cells (Supplementary Figure S3A). The results indicated that the sphere cells display several attributes of prostate CSCs (Supplementary Figure S3B).

\section{CSCs display significant resistance to traditional antitumor drugs} The cell stability assay was used to investigate whether the DU145 sphere cells acquired chemo-resistance. The results showed that the DU145 sphere cells were significantly resistant to cytotoxic chemotherapy, including 5-FU, cis-platinum (DDP) and doxorubicin (Figure 3). Nevertheless, unlike the traditional antitumor drugs, the oncolytic virus GD55 and ZD55, which had previously demonstrated efficacious cancer- killing properties, exerted a strong cytotoxic effect on DU145 sphere cells (Figure 3) without observable resistance.

The data further indicated that some of the prostate cancer cell lines (such as DU145) cultured as tumorspheres in suspension acquired CSC-like properties, which confer resistance to conventional antitumor agents, but retain sensitivity to GD55 and ZD55. Thus, it is plausible that GD55 and ZD55 might be more cytotoxic than traditional chemotherapeutic anti-cancer agents.

\section{GOLPH2-regulated oncolytic adenovirus exhibits elevated} cytotoxic effects on DU145 sphere cells

Previous results have indicated that GOLPH2 is highly expressed in prostate cancer cells. Furthermore, it has been demonstrated that GOLPH2-regulated oncolytic adenovirus exerts potent antitumor effects on prostate cancer cells. Our goal was to examine whether GD55 could effectively eliminate prostate CSCs, such as the sphere cells, in the same manner as previously reported for liver cancer cells.

The cytotoxic effect of GD55 and ZD55 was examined on sphere and parental DU145 cells at the indicated MOIs. The results showed higher E1A protein levels and stronger cytotoxicity in the GD55-treated group compared with those in the ZD55-treated group (Figure 4A and 4B), implying the superiority of GD55. Furthermore, MTT assay was used to test the survival rate of the sphere cells after they were treated with GD55 and ZD55. The cytotoxic effect of GD55 on DU145 

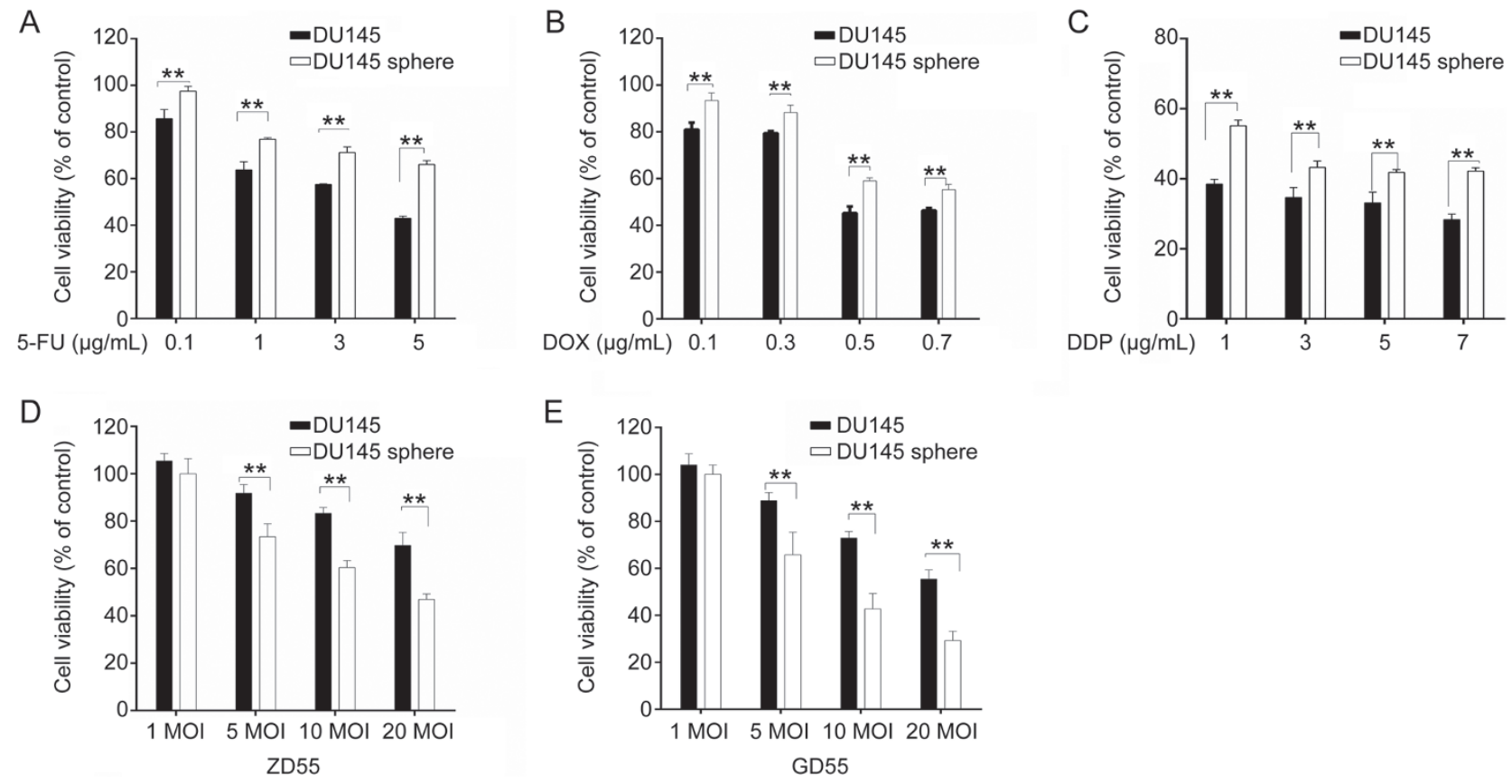

Figure 3. Prostate CSCs displayed significant resistance to traditional antitumor drugs. (A, B and C) After DU145 sphere cells and DU145 parental cells were treated with 5-FU, DDP or doxorubicin (DOX) for 2 days, the DU145 sphere cells displayed more obvious resistance to cytotoxic chemotherapy than DU145 cells. The relative cell viability is shown as fold change to the corresponding mock-treated cells. (D and E) The cytotoxic effects of ZD55 and GD55 on both DU145 sphere cells and parental cells. Cell survival was determined using the MTT assay. The results are expressed as the percentage relative to the mock-treated cells. All experiments were repeated three times, and all data shown represent the mean \pm SD $(n=6)$. ${ }^{*} P<0.05,{ }^{* *} P<0.01$.

sphere cells was more pronounced than that of ZD55 at various time points and MOIs (Figure 4C). The results showed that GD55 exerted a more pronounced proliferation inhibitory effect on prostate CSCs and increasingly exhibited greater cytotoxicity against DU145 sphere cells with time compared with the corresponding effects observed with ZD55 and PBS control.

Further study was conducted to investigate whether GD55 could induce a higher rate of apoptosis in DU145 sphere cells compared with ZD55. Apoptosis was first assessed using the Hoechst 33342 staining assay, which revealed that the fraction of cells with nuclear fragmentation was elevated as expected after the DU145 sphere cells were treated for $48 \mathrm{~h}$ with GD55 compared with that seen in response to ZD55 (Figure 5A). Annexin V staining was used to evaluate the percentage of apoptotic cells. Figure 5B shows the DU145 sphere cells infected with GD55 had a higher percentage of apoptotic cells (1 MOI: $26.4 \%$; $10 \mathrm{MOI}$ : 44.5\%), while a lower percentage of cell apoptosis was observable in the DU145 sphere cells treated with ZD55 (1 MOI: 11.9\%; 10 MOI: 29.7\%) (Figure 5B). Additionally, there were lower levels of anti-apoptosis proteins such as XIAP and Procaspase-3 and higher levels of the cleaved proteins, such as Bax and PARP, as revealed by Western blotting in the GD55-treated DU145 sphere cells, indicating that GD55 exerts more cytotoxic effects than ZD55 (Figure 5C).

Antitumor effect of GOLPH2-mediated GD55 in prostate cancer sphere cells in vivo

The data demonstrating the cytotoxic effect of GD55 on pros- tate CSCs in vitro does not shed light on whether GD55 plays a similar role in vivo, especially on DU145 sphere cells. Therefore, a xenograft model was established in nude mice using DU145 sphere cells to test the tumor-suppressive capacity of GD55. The data showed that xenograft tumors infected with GD55 demonstrated the slowest growth rate, compared with those treated with PBS or infected with ZD55 (Figure 6A). Moreover, histopathological analysis using HE staining showed that GD55 induced higher levels of cell death and necrosis within the tumor mass than ZD55. GD55 did not cause any obvious damage to the prostate tissue, producing the same outcomes as those seen in the PBS-treated control group (Figure 6B). Apoptotic assay via TUNEL revealed similar results. GD55 induced higher rates of apoptosis in tumor cells compared with ZD55 (Figure 6B). These differences in vivo suggested that GD55 is more potent at inhibiting tumors derived from DU145 sphere cells and thus has an enhanced tumor-suppressive effect compared with that of the traditional oncolytic adenovirus ZD55.

\section{Discussion}

The present data suggest that the GOLPH2-regulated oncolytic adenovirus GD55 has potent cytotoxic effects on prostate CSClike cells. ZD55 is a previously constructed oncolytic adenovirus that has been widely reported for its effective cancerkilling ability. We further modified this construct into a novel oncolytic adenovirus GD55, in which the GOLPH2 promoter was utilized to enhance the specificity and high efficiency of 
A

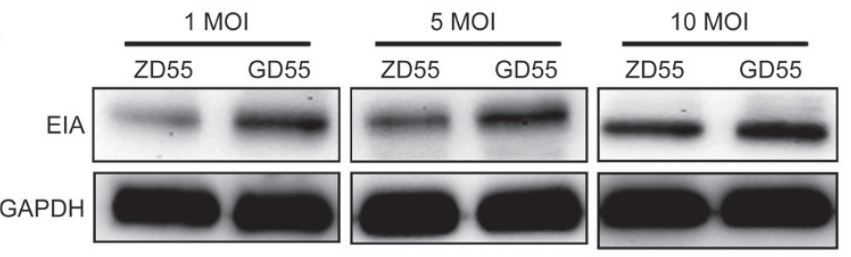

B
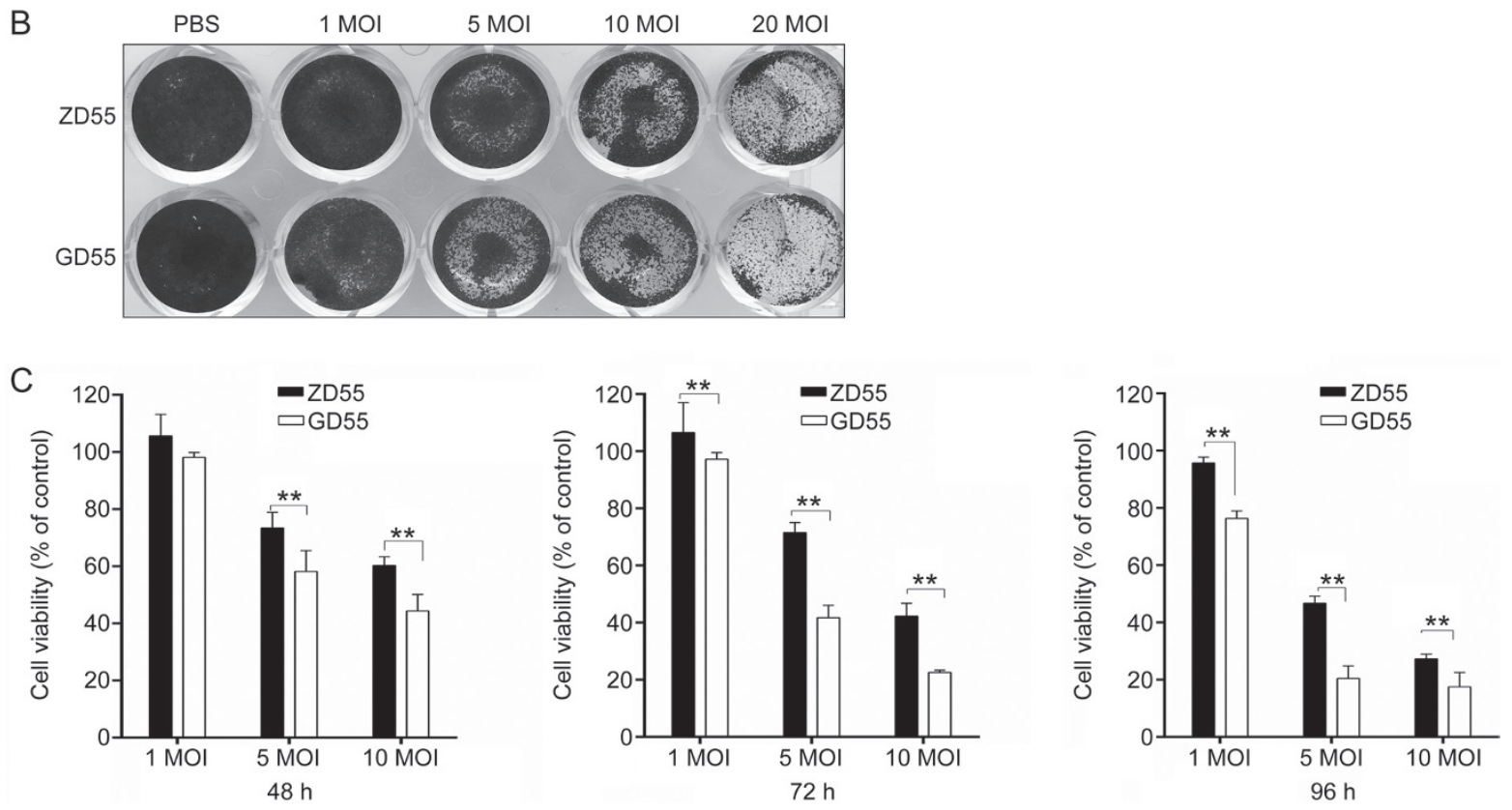

Figure 4. Analysis of infection efficiency and cytotoxicity of GOLPH2-modified adenoviruses on DU145 sphere cells. (A) E1A expression was detected in DU145 sphere cells after treatment with ZD55 and GD55 for 2 days at MOls of 1, 5 and 10. (B) GD55 showed enhanced cytotoxicity in DU145 sphere cells. DU145 sphere cells were treated with the indicated MOI (1, 5, 10 and 20) of ZD55 and GD55 for 2 days and subjected to crystal violet staining for the determination of cell viability. (C) Comparison of cell viability of DU145 sphere cells treated with ZD55 and GD55 at the indicated MOI for 48, 72 and $96 \mathrm{~h}$. All experiments were repeated three times, and all data shown represent the mean $\pm \operatorname{SD}(n=6)$. ${ }^{* *} P<0.01$.

replication of GD55. We have previously tested the efficacy of GD55 in liver CSC-like cells, which demonstrated a higher cytotoxic efficacy than that of ZD55, which was consistent with that exerted in prostate CSC-like cells.

CSCs can self-renew and undergo asymmetric division to promote the growth of the tumor mass, which is responsible for tumor recurrence and can give rise to high metastasis rates, drug-resistance and tumorigenicity ${ }^{[25]}$. Based on this, we successfully enriched for and examined prostate CSC-like cells derived in vitro using suspension cultures as previously described $^{[26]}$. Our experiments showed that the sphere cells have enhanced expression of stemness-associated genes, such as NANOG, OCT4, SOX2, which have been shown to play central roles in the self-renewal and/or development of prostate $\mathrm{CSCS}^{[27-30]}$. Moreover, compared with parental cells, the sphere cells, especially those derived from DU145, expressed high levels of prostate CSC markers such as CD133 and CD44 and phosphorylated AKT, which are associated with the development of prostate cancer ${ }^{[31]}$. Consistent with CSC-like features, compared with parental cells, the DU145 sphere cells showed higher tumorigenicity and proliferative ability in BALB/c mice and were resistant to conventional drugs, but they were sensitive to GD55 and ZD55.

Traditional treatment regimens fail to effectively eradicate CSCs within a tumor. The primary reason for this is that CSCs are typically resistant to conventional drugs, due to their ability to expel small molecules ${ }^{[10]}$. Conversely, adenoviruses, especially oncolytic viruses, cannot be pumped out of CSCs, as they enter CSCs through infection mechanisms, implying their potential for application in tumor therapy. This phenomenon was also verified in our study, as evidenced by the strong cytotoxic effect (Figure 3) and high E1A expression (Figure 4A) in GD55-infected prostate CSC-like cells. GD55 had a better killing effect on CSC-like cells both in cell culture (Figure $4 \mathrm{~B}$ ) and in a mouse model (Figure 6A) due to the superior targeting ability of GOLPH2 compared with ZD55. Notably, the data showed that GD55 induced apoptosis in sphere cells in vitro and in vivo (Figures 5 and 6B), which is consistent with the reports of some of the studies on other cancer-targeting oncolytic adenoviruses ${ }^{[32]}$.

Although GD55 demonstrated significant antitumor effects in our study, there are some limitations to be addressed before GD55 can be further used in clinical trials for prostate cancer, especially when it is delivered via systemic administration. 

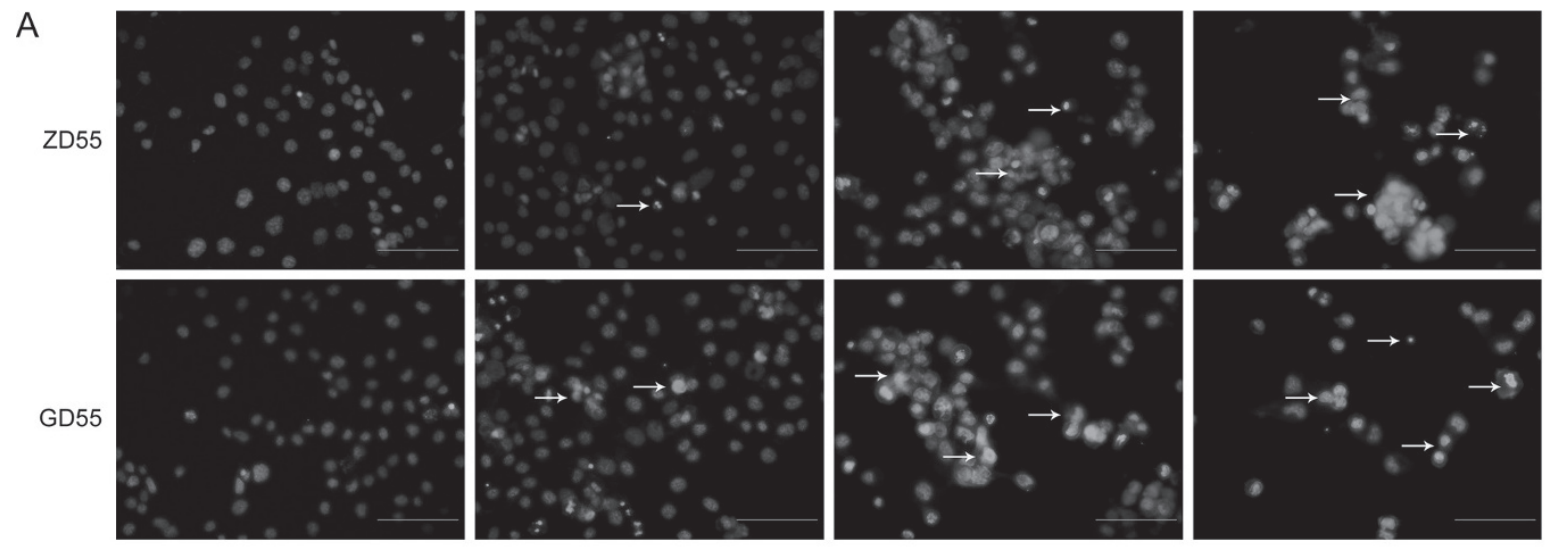

B

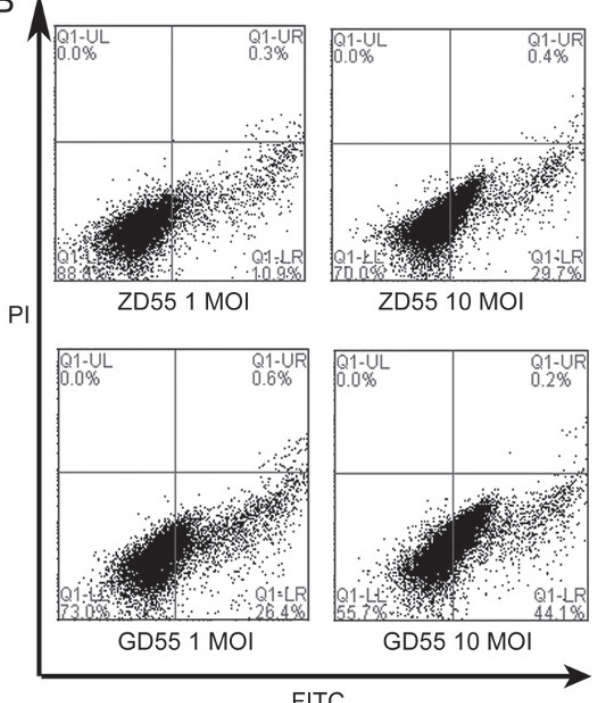

C

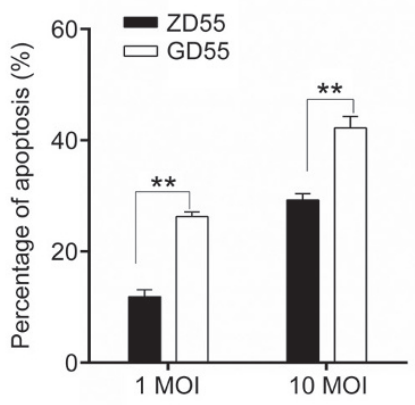

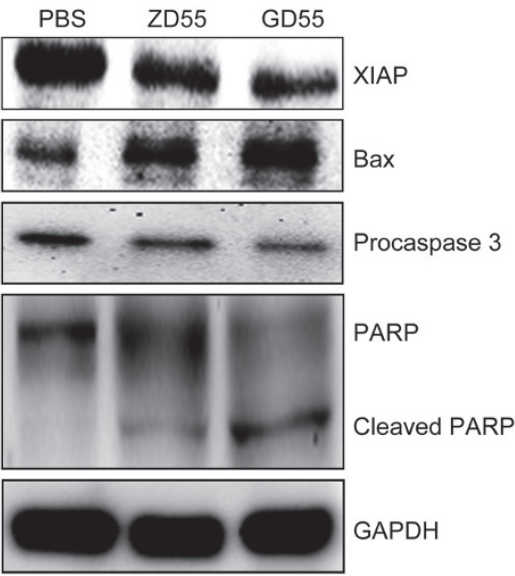

Figure 5. GD55 exhibits marked cytotoxic effects on DU145 sphere cells. (A) Increased nuclear fragmentation (arrow) was observed in DU145 sphere cells after $48 \mathrm{~h}$ of treatment with ZD55 or GD55 at MOls of 1, 5, 10 and 20 as detected by Hoechst staining. Scale bar=200 $\mu$ m. (B) DU145 sphere cells were infected with ZD55 or GD55 at MOI of 1 or 10, and $48 \mathrm{~h}$ later, the cells were stained with Annexin V-FITC and immediately followed by flow cytometry for apoptosis detection. Each value represents the mean of three replicates. ${ }^{* *} P<0.01$. (C) Western blot analysis of the expression of apoptosis-associated proteins. GAPDH was used as an internal control.

The existing limitations include accumulation in the liver and the clearance of viral particles by neutralizing antibodies, immune responses against the virus, and the influence of the tumor microenvironment on viral replication. Currently, these obstacles may be overcome through combinatorial strategies with chemoradiotherapy, immunotherapy with a mechanistic rationale, packaging or modification via nanoparticles or the use of engineered cells as potent vectors for virus delivery. In addition, the capability of GD55 to target prostate CSCs could be improved through further genetic engineering. For example, GD55 can be armed with antitumor elements such as prostate CSC-specific inhibitory genes, RNAi for key transcription factors such as NANOG or the central signaling nodes, such as $\mathrm{AKT} / \mathrm{PI} 3 \mathrm{~K}$, and immune regulation via GM-CSF. These strategies can promote systemic immune responses against tumors and, together with TRAIL or IL-24, effectively eliminate cancer cells through the induction of apoptosis, thereby playing an important role in killing not only CSCs but also non-CSCs within the same tumor ${ }^{[33]}$. Moreover, GOLPH2, as a novel tumor biomarker, has been found during the development of certain other tumor types, eg, liver ${ }^{[16]}$ and gastric tumors ${ }^{[34]}$, implying that GD55 may exert more extensive cytotoxic effects on these tumors.

Recent reports have revealed that an interesting plasticity exists between non-CSCs and CSCs in breast carcinomas ${ }^{[35]}$. These studies show that CSCs are able to give rise to non-CSC progeny, and vice versa, in which more differentiated nonCSCs can readily convert to a CSC-like state. It is plausible that this plasticity robustly drives the metastatic dissemination of neoplastic cells and causes disease relapse, not only in breast cancer but also in other types of cancer. These and other observations suggest that GD55 might be a good candidate for the treatment of prostate cancer and other types of carcinomas. 


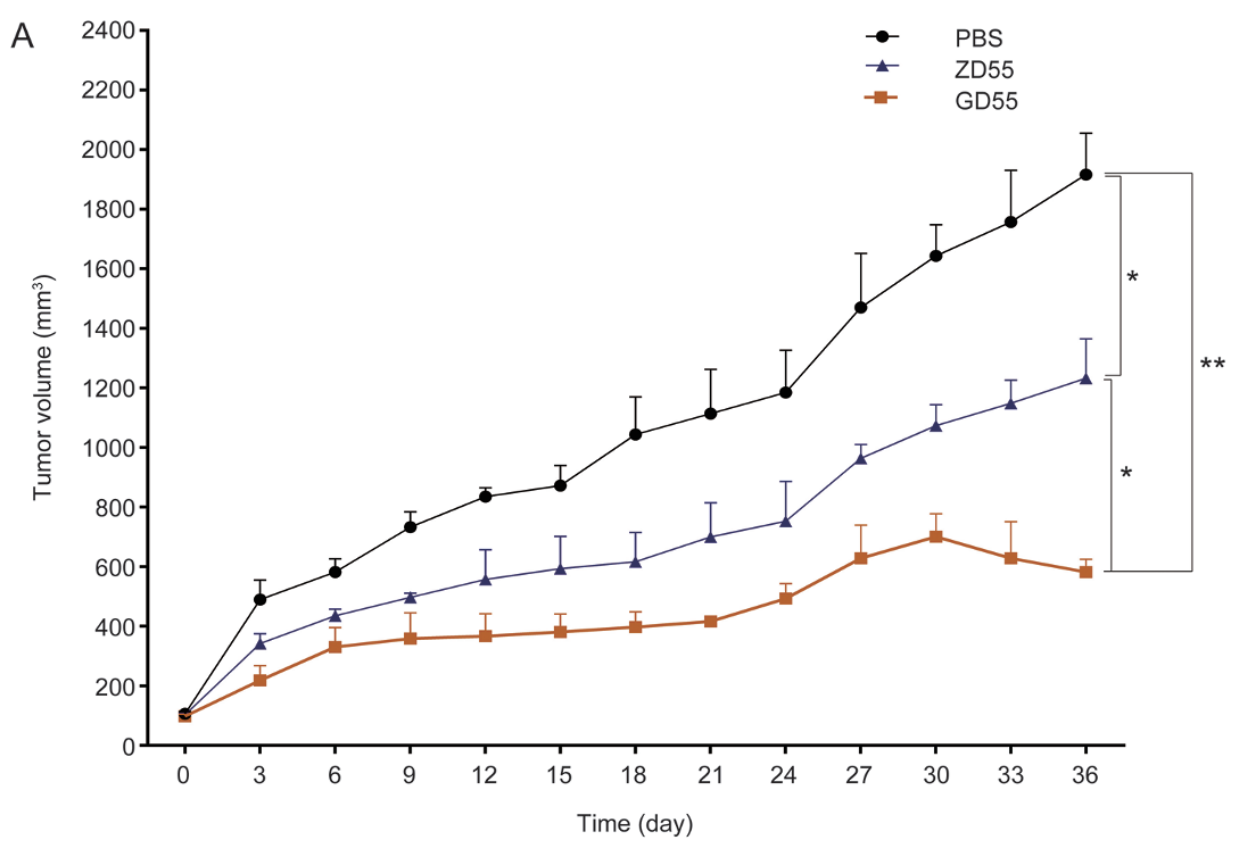

B
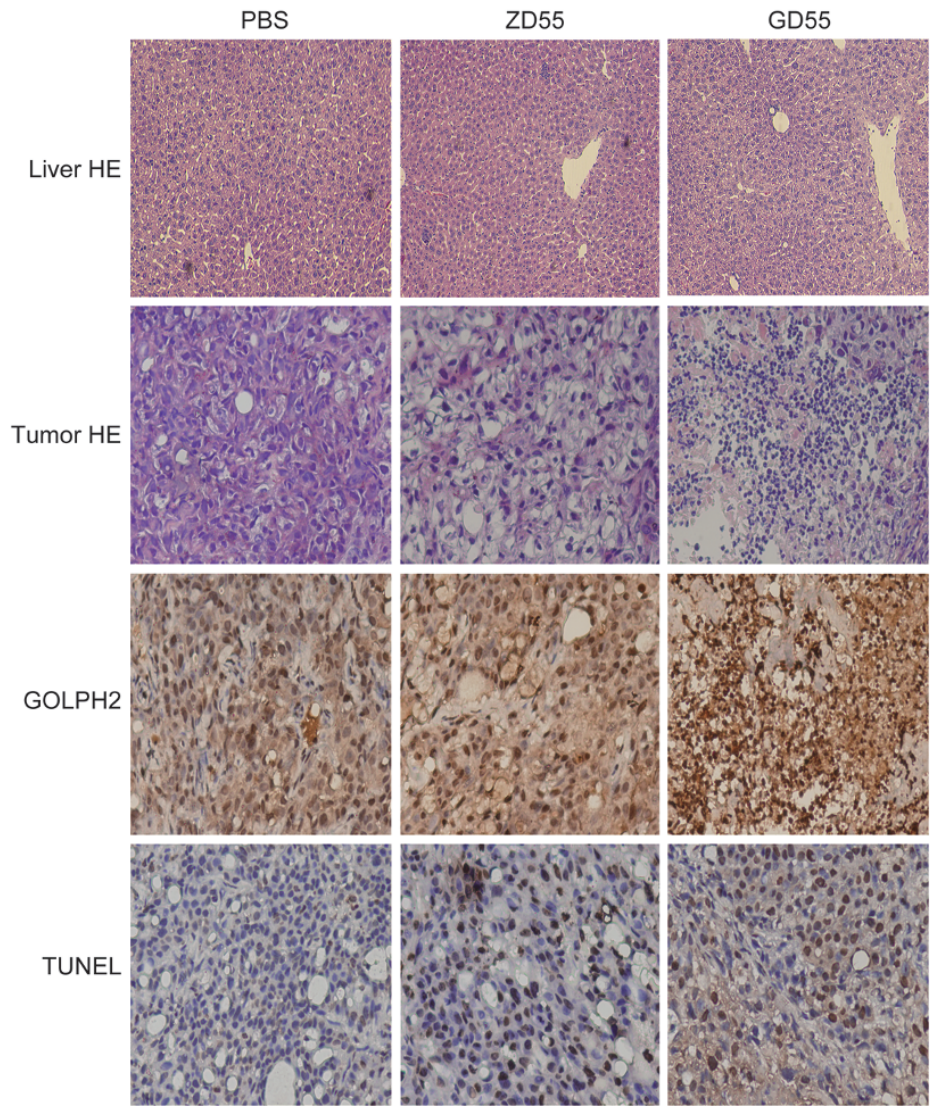

Figure 6. Antitumor effect of GD55 on prostate cancer sphere cells in vivo. (A) DU145 sphere cells were subcutaneously inoculated into female BALB/c nude mice at $1.5 \times 10^{6}$ cells per mouse to generate tumor xenografts. When the tumor volume reached $100 \mathrm{~mm}^{3}$, the mice were randomly divided into three groups $(n=8)$ and infected with $1 \times 10^{9}$ PFU of ZD55, GD55 or PBS every other day, and the infection was repeated three times. The tumor volumes were monitored by periodic measurement. Tumor volume $(\mathrm{V})$ was calculated according the formula $\left.\mathrm{V}(\mathrm{mm})^{3}\right)=1 \mathrm{ength}(\mathrm{mm}) \times \mathrm{width}(\mathrm{mm})^{2} / 2$. (B) Histological analysis of tumor sections in the PBS, ZD55 and GD55 groups of DU145 sphere xenograft tumors. The upper two rows represent hematoxylin and eosin (H\&E) stained images of animal liver and tumor tissues, indicating hepatotoxicity and necrotic areas in the tumors. The middle row represents GOLPH2 expression by IHC analysis in tumor tissues. The lower row represents TUNEL assay for detecting apoptotic cells in the tumor tissues treated with the various agents. GD55 induced more apoptosis of tumor cells. The brown color represents the apoptotic cells (original magnification: $\times 200)$. ${ }^{*} P<0.05,{ }^{* *} P<0.01$. 


\section{Author contribution}

Chang YING and Yi-gang WANG designed the study; Xinyuan LIU, Yi-gang WANG and Hui YAN led the study and edited the manuscript; Chang YING, Bo-duan XIAO and Yun QIN performed the experiments; Chang YING and Bo-duan XIAO analyzed the data and wrote the manuscript; and Binrong WANG, Ru-wei WANG, Ling FANG and Xiu-mei ZHOU assisted in performing the experiments.

\section{Acknowledgements}

This work was supported by the National Natural Science Foundation of China (№ 81272687 and 81573000), Zhejiang Provincial Natural Science Foundation of China (№ LY16H160056), Zhejiang Province Health Department (№ 2014RCA022), Grant for 521 Talent Project of ZSTU and Academician Workstation of Zhejiang Province.

\section{Supplementary information}

Supplementary information is available at the website of Acta Pharmacologica Sinica.

\section{References}

1 Holzbeierlein JM. Long-term survival after radical prostatectomy versus external-beam radiotherapy for patients with high-risk prostate cancer. Cancer 2011; 117: 2830-2.

2 Rajasekhar VK, Studer L, Gerald W, Socci ND, Scher HI. Tumourinitiating stem-like cells in human prostate cancer exhibit increased NF-kappaB signalling. Nat Commun 2011; 2: 162.

3 Magee JA, Piskounova E, Morrison SJ. Cancer stem cells: impact, heterogeneity, and uncertainty. Cancer Cell 2012; 21: 283-96.

4 Visvader JE, Lindeman GJ. Cancer stem cells: current status and evolving complexities. Cell Stem Cell 2012; 10: 717-28.

5 Meacham CE, Morrison SJ. Tumour heterogeneity and cancer cell plasticity. Nature 2013; 501: 328-37.

6 Leong KG, Wang BE, Johnson L, Gao WQ. Generation of a prostate from a single adult stem cell. Nature 2008; 456: 804-8.

7 Yu DB, Zhong SY, Yang M, Wang YG, Qian QJ, Zheng S, et al. Potent antitumor activity of double-regulated oncolytic adenovirus-mediated ST13 for colorectal cancer. Cancer Sci 2009; 100: 678-83.

8 Zhang KJ, Wang YG, Cao X, Zhong SY, Wei RC, Wu YM, et al. Potent antitumor effect of interleukin-24 gene in the survivin promoter and retinoblastoma double-regulated oncolytic adenovirus. Hum Gene Ther 2009; 20: 818-30.

9 Cripe TP, Wang PY, Marcato P, Mahller YY, Lee PW. Targeting cancerinitiating cells with oncolytic viruses. Mol Ther 2009; 17: 1677-82.

10 Dean M, Fojo T, Bates S. Tumour stem cells and drug resistance. Nat Rev Cancer 2005; 5: 275-84.

11 Yang Y, Xu H, Huang W, Ding M, Xiao J, Yang D, et al. Targeting lung cancer stem-like cells with TRAIL gene armed oncolytic adenovirus. J Cell Mol Med 2015; 19: 915-23.

12 Zhao L, Gu J, Dong A, Zhang Y, Zhong L, He L, et al. Potent antitumor activity of oncolytic adenovirus expressing mda-7/IL-24 for colorectal cancer. Hum Gene Ther 2005; 16: 845-58.

13 Liu XY, Gu JF. Targeting gene-virotherapy of cancer. Cell Res 2006; 16: 740.

14 Zhang ZL, Zou WG, Luo CX, Li BH, Wang JH, Sun LY, et al. An armed oncolytic adenovirus system, ZD55-gene, demonstrating potent antitumoral efficacy. Cell Res 2003; 13: 481-9.

15 Zhang Y, Gu J, Zhao L, He L, Qian W, Wang J, et al. Complete elimination of colorectal tumor xenograft by combined manganese superoxide dismutase with tumor necrosis factor-related apoptosisinducing ligand gene virotherapy. Cancer Res 2006; 66: 4291-8.
16 Wang Y, Liu T, Huang P, Zhao H, Zhang R, Ma B, et al. A novel Golgi protein (GOLPH2)-regulated oncolytic adenovirus exhibits potent antitumor efficacy in hepatocellular carcinoma. Oncotarget 2015; 6: 13564-78.

17 Zhang X, Meng S, Zhang R, Ma B, Liu T, Yang Y, et al. GP73-regulated oncolytic adenoviruses possess potent killing effect on human liver cancer stem-like cells. Oncotarget 2016; 7: 29346-58.

18 Yang $\mathrm{Y}, \mathrm{Xu} \mathrm{H}$, Shen J, Wu S, Xiao J, Xu Y, et al. RGD-modifided oncolytic adenovirus exhibited potent cytotoxic effect on CAR-negative bladder cancer-initiating cells. Cell Death Dis 2015; 14: 128.

19 Collins AT, Berry PA, Hyde C, Stower MJ, Maitland NJ. Prospective identification of tumorigenic prostate cancer stem cells. Cancer Res 2005; 65: 10946-51.

20 Collura A, Marisa L, Trojan D, Buhard O, Lagrange A, Saget A, et al. Extensive characterization of sphere models established from colorectal cancer cell lines. Cell Mol Life Sci 2013; 70: 729-42.

21 Singh SK, Clarke ID, Terasaki M, Bonn VE, Hawkins C, Squire J, et al. Identification of a cancer stem cell in human brain tumors. Cancer Res 2003; 63: 5821-8.

22 Ding BS, James D, lyer R, Falciatori I, Hambardzumyan D, Wang S, et al. Prominin 1/CD133 endothelium sustains growth of proneural glioma. PLoS One 2013; 8: 1093-7.

23 Missol-Kolka E, Karbanova J, Janich P, Haase M, Fargeas CA, Huttner WB, et al. Prominin-1 (CD133) is not restricted to stem cells located in the basal compartment of murine and human prostate. Prostate 2011; 71: 254-67.

24 Hurt EM, Kawasaki BT, Klarmann GJ, Thomas SB, Farrar WL. CD44 ${ }^{+}$ CD24(-) prostate cells are early cancer progenitor/stem cells that provide a model for patients with poor prognosis. Br J Cancer 2008; 98: 756-65.

25 Patrawala L, Calhoun T, Schneider-Broussard R, Li H, Bhatia B, Tang $\mathrm{S}$, et al. Highly purified $\mathrm{CD} 44^{+}$prostate cancer cells from xenograft human tumors are enriched in tumorigenic and metastatic progenitor cells. Oncogene 2006; 25: 1696-708.

26 Wang L, Huang X, Zheng X, Wang X, Li S, Zhang L, et al. Enrichment of prostate cancer stem-like cells from human prostate cancer cell lines by culture in serum-free medium and chemoradiotherapy. Int J Biol Sci 2013; 9: 472-9.

27 Gu G, Yuan J, Wills M, Kasper S. Prostate cancer cells with stem cell characteristics reconstitute the original human tumor in vivo. Cancer Res 2007; 67: 4807-15.

28 Jeter CR, Badeaux M, Choy G, Chandra D, Patrawala L, Liu C, et al. Functional evidence that the self-renewal gene NANOG regulates human tumor development. Stem Cells 2009; 27: 993-1005.

29 Jeter CR, Liu B, Liu X, Chen X, Liu C, Calhoun-Davis T, et al. NANOG promotes cancer stem cell characteristics and prostate cancer resistance to androgen deprivation. Oncogene 2011; 30: 3833-45.

30 Ugolkov AV, Eisengart LJ, Luan C, Yang XJ. Expression analysis of putative stem cell markers in human benign and malignant prostate. Prostate 2011; 71: 18-25.

31 Dubrovska A, Kim S, Salamone RJ, Walker JR, Maira SM, GarciaEcheverria C, et al. The role of PTEN/Akt/PI3K signaling in the maintenance and viability of prostate cancer stem-like cell populations. Proc Natl Acad Sci U S A 2009; 106: 268-73.

32 Thaci B, Ulasov IV, Ahmed AU, Ferguson SD, Han Y, Lesniak MS. Antiangiogenic therapy increases intratumoral adenovirus distribution by inducing collagen degradation. Gene Ther 2013; 20: 318-27.

33 Liu XY. The excellent anti-tumour strategy (CTGVT, OV-gene) and the excellent anti-tumor gene (IL-24). Int J Biomed Sci 2012; 8: 87-93.

34 Chen LG, Wang HJ, Yao HB, Guan TP, Wu F, He XJ, et al. GP73 is down-regulated in gastric cancer and associated with tumor differentiation. World J Surg Oncol 2013; 11: 132.

35 Chaffer CL, Marjanovic ND, Lee T, Bell G, Kleer CG, Reinhardt F, et al. Poised chromatin at the ZEB1 promoter enables breast cancer cell plasticity and enhances tumorigenicity. Cell 2013; 154: 61-74. 